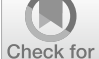

Check for

updates

Cite as

Nano-Micro Lett.

(2021) 13:179

Received: 27 April 2021

Accepted: 25 July 2021

Published online: 18 August 2021

(C) The Author(s) 2021

\section{In Situ Monitoring the Potassium-Ion Storage Enhancement in Iron Selenide with Ether-Based Electrolyte}

\author{
Xiaodan $\mathrm{Li}^{1}$, Jinliang $\mathrm{Li}^{1}{ }^{凶}$, Wenchen Zhuo ${ }^{1}$, Zhibin $\mathrm{Li}^{1}$, Liang $\mathrm{Ma}^{1}$, Zhong $\mathrm{Ji}^{1}$, \\ Likun Pan $^{2}$, Wenjie Mai ${ }^{1} \bowtie$
}

\title{
HIGHLIGHTS
}

- The iron selenide composite with ether-based electrolyte presents excellent potassium storage performance.

- We develop in situ visualization technique to monitor the potassiation-depotassiation process.

- Iron selenide composite in ether-based electrolyte presents a homogeneous electrochemical reaction, resulting in a stable potassiumion storage.

\begin{abstract}
As one of the promising anode materials, iron selenide has received much attention for potassium-ion batteries (KIBs). Nevertheless, volume expansion and sluggish kinetics of iron selenide result in the poor reversibility and stability during potassiation-depotassiation process. In this work, we develop iron selenide composite matching ether-based electrolyte for
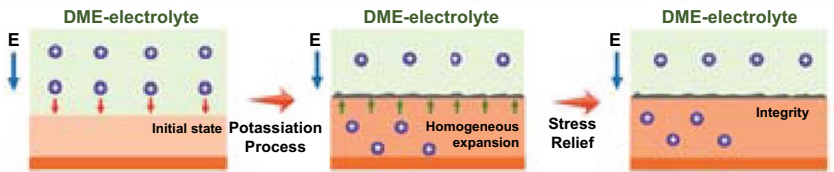

$\longrightarrow$ Ion migration path

$\longrightarrow$ Force direction

o K-ion

DME-electrolyte

EP-electrolyte

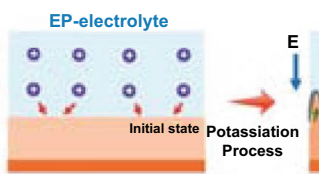

EP-electrolyte

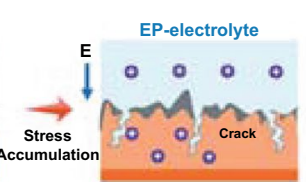

SEI layer

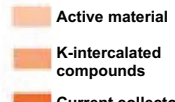
KIBs, which presents a reversible specific capacity of $356 \mathrm{mAh} \mathrm{g}^{-1}$ at $200 \mathrm{~mA} \mathrm{~g}^{-1}$ after 75 cycles. According to the measurement of mechanical properties, it is found that iron selenide composite also exhibits robust and elastic solid electrolyte interphase layer in ether-based electrolyte, contributing to the improvement in reversibility and stability for KIBs. To further investigate the electrochemical enhancement mechanism of ether-based electrolyte in KIBs, we also utilize in situ visualization technique to monitor the potassiation-depotassiation process. For comparison, iron selenide composite matching carbonate-based electrolyte presents vast morphology change during potassiation-depotassiation process. When changing to ether-based electrolyte, a few minor morphology changes can be observed. This phenomenon indicates an occurrence of homogeneous electrochemical reaction in ether-based electrolyte, which results in a stable performance for potassium-ion (K-ion) storage. We believe that our work will provide a new perspective to visually monitor the potassium-ion storage process and guide the improvement in electrode material performance.
\end{abstract}

KEYWORDS Iron selenide; Ether-based electrolyte; In situ visualization technique; Potassium-ion batteries

Jinliang Li, lijinliang@email.jnu.edu.cn; Wenjie Mai, wenjiemai@email.jnu.edu.cn

1 Siyuan Laboratory, Guangzhou Key Laboratory of Vacuum Coating Technologies and New Energy Materials, Guangdong Provincial Engineering Technology Research Center of Vacuum Coating Technologies and New Materials, Department of Physics, Jinan University, Guangzhou, Guangdong 510632, People's Republic of China

2 Shanghai Key Laboratory of Magnetic Resonance, School of Physics and Electronic Science, East China Normal University, Shanghai 200241, People's Republic of China 


\section{Introduction}

Since commercialization, lithium-ion batteries (LIBs) have developed rapidly and gained a monopoly position in portable device and electric vehicle industry due to the handiness and high energy density [1-4]. However, scarce reserves and maldistribution of lithium resource restrict the further development of LIBs in large-scale energy system [5-7]. Exploring low-cost energy storage system becomes urgent. As one of the promising alternatives, potassiumion batteries (KIBs) have been regarded as an ideal energy storage system to replace LIBs due to the similar properties to lithium and abundant potassium resources [8-10]. Despite these advantages, the electrode materials usually accommodate large volume variations during the repetitive potassiation-depotassiation process due to the large size of potassium (K)-ion, which results in the irreversible behavior and degraded cycling stability $[11,12]$. Therefore, seeking suitable host materials with high reversibility and stability for K-ion storage becomes an important research direction.

Because of the low cost and stability, enormous efforts are mainly focused on the carbonaceous materials, which have become the primary selectivity for KIBs [13-15]. However, low theoretical capacities of carbonaceous materials restrict the further improvement in the $\mathrm{K}$-ion storage performance, forcing scientists to explore other highperformance host materials $[16,17]$. Due to the superior $\mathrm{K}$-ion storage performance, iron selenide also obtains lots of attention [18, 19], whereas enormous volume variations also persecute the improvement in the cycling stability for KIBs [20, 21]. Designing smart structures of iron selenide becomes an important path to address the issue of volume variations, which is helpful for the improvement in stability of KIBs. Liu et al. designed iron selenide anchored on nitrogen-doped carbon via an in situ chemical transformation method, achieving a reversible specific capacity of 434 $\mathrm{mAh} \mathrm{g}^{-1}$ after 70 cycles at $100 \mathrm{~mA} \mathrm{~g}^{-1}$ for KIBs [22]. Zhao et al. synthesized iron selenide nanodots porous carbon networks by a gas-pumped self-expanding method, presenting a reversible specific capacity of $330 \mathrm{mAh} \mathrm{g}^{-1}$ after 100 cycles at $100 \mathrm{~mA} \mathrm{~g}^{-1}$ for K-ion storage [23]. Wang et al. obtained dispersed iron selenide nanoparticles in porous carbon nanofibers via a multistep hydrothermal method, retaining a decent charge capacity of $201 \mathrm{mAh} \mathrm{g}^{-1}$ after 50 cycles at $100 \mathrm{~mA} \mathrm{~g}^{-1}$ for KIBs [24]. Although the above work delivered the excellent K-ion storage performance, the complex preparation process is still unsatisfactory for the current demand of KIBs. Exploring simple methods to improve the electrochemical performance of iron selenide is urgent.

As another effective strategy, optimizing electrolyte can also remarkably enhance the K-ion storage performance of electrode [25-27]. Currently, ether-based electrolytes have been demonstrated to facilitate the formation of robust solid electrolytes interface (SEI) layer, which can provide a favorable working environment for $\mathrm{K}$-ion transportation and contribute to the cycling stability. Wu et al. found that dimethyl ether electrolyte could hold the interface stability of $\mathrm{SnSb} / \mathrm{C}$ anodes against large volume changes, eventually realizing an excellent cyclability [28]. Lei et al. obtained Bi anode cooperated with ether-based electrolyte for K-ion storage, achieving a fast kinetics and high tolerance of volume change, which contributed to the high K-ion storage performance [29]. Han et al. designed $\mathrm{FeS}_{2} @ \mathrm{C}$ composite cooperated with ether-based electrolyte and raised the cutoff voltage, implementing a boosting long-term cycle stability [30]. In spite of these works, more material systems cooperated with ether-based electrolyte still need to be further explored. Meanwhile, due to different reaction environments of electrodes in ether-based electrolytes, some unconventional electrochemical reaction phenomena compared with that in carbonate-based (some named as ester-based) electrolytes occur and the detailed mechanism is still unclear. Utilizing in situ techniques to monitor the electrochemical reaction of electrodes and probing the underlying mechanism in etherbased electrolytes have become necessary [31-33].

In this work, we rationally obtained reduced graphene oxide coating iron selenide ( $\mathrm{FeSe}_{2} @ \mathrm{RGO}$ ) composite by a simple hydrothermal process. After optimization, we found that our $\mathrm{FeSe}_{2} @ \mathrm{RGO}$ composite matching etherbased electrolyte presented a remarkable improvement in electrochemical performance compared with that using the carbonate-based electrolyte. To explore the improvement in electrochemical performance, we also measured the mechanical properties of electrodes in both electrolytes. The result shows that the electrode in the ether-based electrolyte exhibits higher Young's modulus, which can effectively enhance the stability of electrode. Furthermore, the visualization technique was also developed, which could in situ monitor the electrochemical reaction process of electrode. It was 
found that the electrode in ether-based electrolyte presented homogeneous electrochemical reaction, resulting in a stable morphology change during potassiation-depotassiation process, which contributed to the improvement in electrochemical performance. Compared with the vast morphology change of electrode in carbonate-based electrolyte, a few minor changes could be observed, indicating an occurrence of homogeneous electrochemical reaction in ether-based electrolyte, which resulted in a stable performance for K-ion storage.

\section{Experiment}

\subsection{Synthesis}

Typically, GO was obtained by modified Hummers' method from natural graphite, which has been reported in our previous work [34, 35]. For the $\mathrm{FeSe}_{2}$-RGO composites, $1.067 \mathrm{~g}$ $\mathrm{Fe}\left(\mathrm{NH}_{4}\right)_{2}\left(\mathrm{SO}_{4}\right)_{2} \cdot 6 \mathrm{H}_{2} \mathrm{O}$ and $2 \mathrm{~g}$ citric acid were dissolved into $30 \mathrm{~mL}$ deionized water, and then, $30 \mathrm{~mL}$ aqueous solution with $1 \mathrm{~g} \mathrm{NaBH}_{4}$ and $0.431 \mathrm{~g} \mathrm{Se}$ powder was dropped under vigorous stirring. To form homogeneous dispersions, moderate GO was added into above solution under stirring and sonication over $1 \mathrm{~h}$. The mixture was transformed into a $100 \mathrm{~mL}$ Teflon-lined stainless steel autoclave and heated at $180{ }^{\circ} \mathrm{C}$ for $12 \mathrm{~h}$. Subsequently, black precipitate was collected, washed with water for several times and dried under freezer dryer. After that, the black precipitate was annealed at $400{ }^{\circ} \mathrm{C}$ for $3 \mathrm{~h}$ under the flowing nitrogen to obtain the $\mathrm{FeSe}_{2} @ \mathrm{RGO}$ composite. Among them, the composites added 0, 180, 240 and $300 \mathrm{mg}$ GO were named as $\mathrm{FeSe}_{2}$, $\mathrm{FeSe}_{2} @ \mathrm{RGO}-1, \mathrm{FeSe}_{2} @ \mathrm{RGO}$ and $\mathrm{FeSe}_{2} @ \mathrm{RGO}-3$, respectively. Unless otherwise specified, the electrode should be the $\mathrm{FeSe}_{2} @ \mathrm{RGO}$. For comparison, pure RGO was also obtained by the similar methods without the precursor of $\mathrm{FeSe}_{2}$.

\subsection{Characterization}

The morphologies of samples were measured by fieldemission scanning electron microscopy (FESEM, Zeiss) and transmission electron microscopy (TEM, FEI). The structures were confirmed by X-ray diffraction (XRD, D8 Rigaku9000) and Raman spectroscopy (Horiba T6400). The surface features were recorded by X-ray photoelectron spectroscopy (XPS, Thermo Fisher Scientific, K-Alpha). For the in situ visualization observation, we used metallographic microscope (Leica) cooperated with an electrochemical reaction cell to realize the in situ observation of electrode.

\subsection{Electrochemical Measurement}

The as-prepared samples were used as the anode materials of KIBs to measure the electrochemical performance. The active material, super $\mathrm{P}$ and carboxylmethyl cellulose were mixed in the weight ratio of 8:1:1 in deionized water to form a uniform slurry. After that, the slurry was covered on $\mathrm{Cu}$ foil and dried overnight at $80{ }^{\circ} \mathrm{C}$ in a vacuum oven. Generally, the mass loading of above electrode was about $1.0-1.5 \mathrm{mg} \mathrm{cm}^{-2}$ and was acted as work electrode in CR2032 coin cells, which were assembled in argon-filled Etelux glovebox (Lab 2000) with the water and oxygen content less than $0.1 \mathrm{ppm}$. The $\mathrm{K}$ metal foil and Whatman glass fiber membrane were served as counter electrode and separator, respectively. The solution of ethylene glycol dimethyl ether with $1 \mathrm{M}$ and $5 \mathrm{M}$ potassium bis(fluorosulfonyl) imide (KFSI) was used as electrolyte, which was named as DME- 1 and DME. For comparison, we also used 1 M KFSI in ethylene carbonate and propylene carbonate $(1: 1, \mathrm{v} / \mathrm{v})$ as electrolyte, which was named as EP. The galvanostatic charge-discharge (GCD) curves and the cycling performance were measured by battery test system (Neware BTS 4000 ) with the voltage ranging from 0.01 to $3 \mathrm{~V}$ at a current density of $100 \mathrm{~mA} \mathrm{~g}^{-1}$ unless otherwise specified. The cyclic voltammetry $(\mathrm{CV})$ curves were recorded by electrochemical workstation (Shanghai ChenHua). The electrochemical impedance spectroscopies (EIS) were measured by Princeton electrochemical workstation (Veras STAT 3400). For the in situ visualization measurement, we used the dual electrode electrolytic tank under argon atmosphere, as shown in Fig. S1. Figure S2 shows the photograph of in situ visualization testing system. The detail assembly process is similar to the K-ion half battery. Firstly, the active material electrode was put in bottom and then placed the separator and metallic $\mathrm{K}$ foil which have been punched. The top was sealed with the quartz glass, which serves as observation window for the visualization. During the measurement, the in situ electrolytic tank was connected with the electrochemical station at a scan rate of $0.6 \mathrm{mV} \mathrm{s}^{-1}$. From the observation window, we could obtain the changes of electrode morphology 
during different potassiation-depotassiation states by optical microscope.

\section{Results and Discussion}

\subsection{Materials Characterization}

Figure 1a shows the SEM image of $\mathrm{FeSe}_{2}$, which exhibits a typical granular structure. With the introduction of RGO in composite, the $\mathrm{FeSe}_{2}$ particles are packaged by RGO nanosheets to form a network structure, as shown in Fig. $1 \mathrm{~b}$. From the TEM image of $\mathrm{FeSe}_{2} @ \mathrm{RGO}$ (Fig. 1c), the package structure of composite is also confirmed. Due to the excellent conductivity and stability of RGO in composite, the package network structure of composite can effectively enhance the electronic transport property and accommodate the volume expansion during potassiation-depotassiation process. Figure 1d shows the high-resolution TEM image of $\mathrm{FeSe}_{2} @ \mathrm{RGO}$. The distinct lattice fringes can be detected, indicating good crystallinity of $\mathrm{FeSe}_{2}$. A lattice distance of $0.29 \mathrm{~nm}$ is observed, which can be indexed to the (101) plane of $\mathrm{FeSe}_{2}$ (PDF No. 79-1892) [36, 37]. Figure 1e-h presents the element mapping of $\mathrm{FeSe}_{2} @ \mathrm{RGO}$ composite. The result shows that $\mathrm{Se}$ and $\mathrm{Fe}$ are distributed uniformly in the $\mathrm{FeSe}_{2}$ particle and the $\mathrm{C}$ is distributed homogeneously in RGO layers. Figure S3a presents the XRD pattern of RGO, $\mathrm{FeSe}_{2}$ and $\mathrm{FeSe}_{2} @ \mathrm{RGO}$. We also found that $\mathrm{FeSe}_{2} @ \mathrm{RGO}$ composite shows the same peaks compared with $\mathrm{FeSe}_{2}$ (PDF No. 79-1892), indicating that the introduction of RGO in composite does not change its structure [21]. No RGO peaks
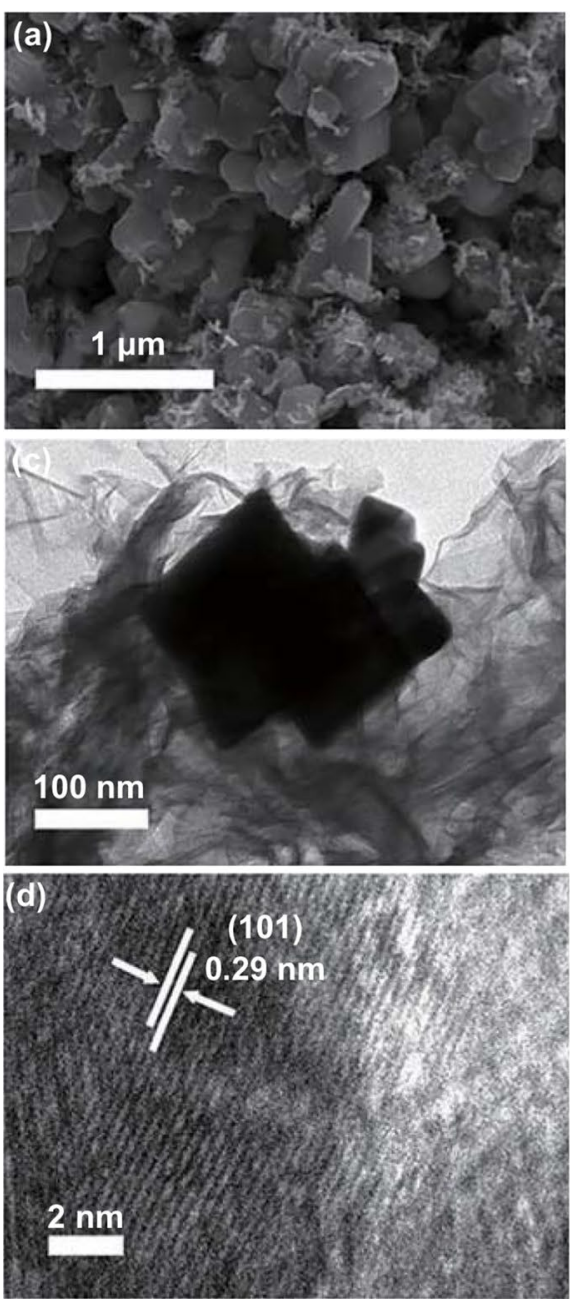
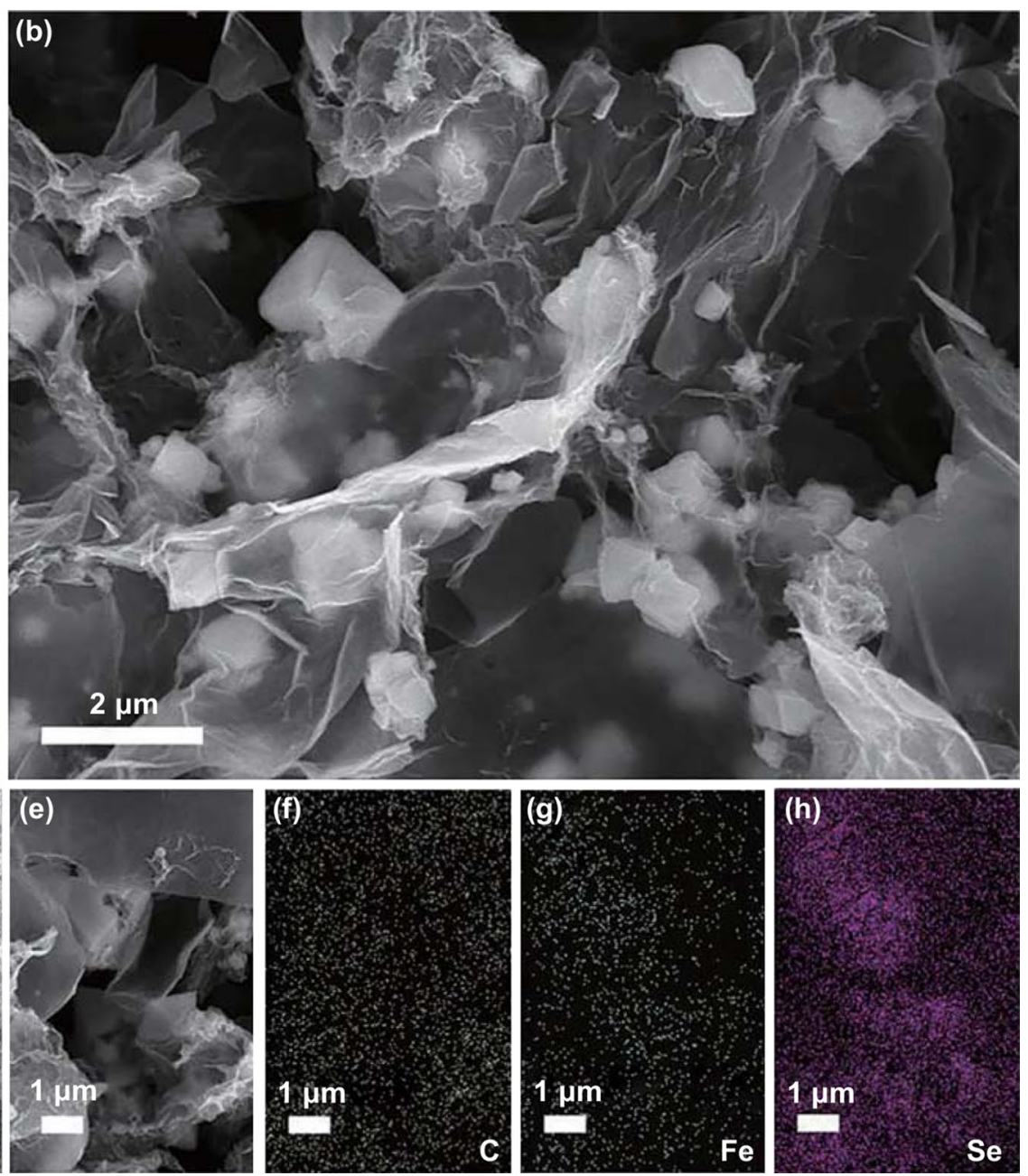

Fig. 1 SEM images of $\mathbf{a ~} \mathrm{FeSe}_{2}$ and $\mathbf{b} \mathrm{FeSe}_{2} @ \mathrm{RGO}$, c low-resolution and $\mathbf{d}$ high-resolution TEM image of FeSe $\mathrm{F}_{2} \mathrm{RGO}$. e-h C, Fe, Se elements mapping of $\mathrm{FeSe}_{2} @ \mathrm{RGO}$ 
appear in composite, which should be due to the weak crystallinity of RGO [38]. To further investigate the structure of $\mathrm{FeSe}_{2} @ \mathrm{RGO}$ composite, the Raman spectrum of FeSe $\mathrm{F}_{2} @$ RGO composite is provided (Fig. S3b). Two Raman peaks at low wavenumbers can be observed, which should be due to the rocking and stretching vibration of Se-Se bonds or their combination [39]. Other two peaks at high wavenumbers should be the $\mathrm{D}$ and $\mathrm{G}$ bands of RGO, further indicating that RGO has been introduced in the composite. To examine the surface property, XPS spectra of our composite were conducted. Figure S3c shows the Fe $2 p$ high-resolution XPS spectrum. Three peaks can be deconvoluted at 707.3, 711.1 and $720.3 \mathrm{eV}$, which are ascribed to the Fe $2 p_{3 / 2}$, satellite peak of $\mathrm{Fe} 2 p_{3 / 2}$ and $\mathrm{Fe} 2 p_{1 / 2}$, respectively [40]. Figure S3d presents the Se $3 d$ high-resolution XPS spectrum, which can be deconvoluted into four peaks. Among them, two peaks at 55.2 and $55.8 \mathrm{eV}$ correspond to Se $3 d_{5 / 2}$ and Se $3 d_{3 / 2}$, respectively [40].

\subsection{Electrochemical Performance Analysis}

Figure S4 shows the cycling performance of RGO, $\mathrm{FeSe}_{2}$, $\mathrm{FeSe}_{2} @ \mathrm{RGO}-1, \mathrm{FeSe}_{2} @ \mathrm{RGO}$ and FeSe $2 @ \mathrm{RGO}-3$, and the results show that $\mathrm{FeSe}_{2} @ \mathrm{RGO}$ exhibits better K-ion storage performance. Based on this, we selected $\mathrm{FeSe}_{2} @ \mathrm{RGO}$ to match different electrolytes for analyzing the effect of electrolyte. To optimize the electrolyte, we also attempt to using DME-1 electrolyte in our electrode for KIBs. Figure S5 shows the corresponding GCD curves. It is found that the battery cannot charge to the set voltage of $3 \mathrm{~V}$, indicating that the DME-1 is inappropriate for KIBs. Figure 2a shows the GCD curves of FeSe ${ }_{2} @$ RGO with DME- and EP-based electrolytes. An irreversible reaction in initial potassiation process can be found in the electrode using both electrolytes. However, the electrode in DME-based electrolyte receives a high reversibility for KIBs, indicating the higher transfer ability of K-ion in DME-based electrolyte. To further investigate the electrochemical reaction, the $\mathrm{CV}$ curves of electrode matching DME-based and EP-based electrolytes are also provided, as shown in Fig. 2b. An obvious irreversible cathodic peak of battery with DME-based electrolyte at $0.77 \mathrm{~V}$ can be observed, which is attributed to the formation of SEI layer. But the irreversible cathodic peak of electrode in EP-based electrolyte exhibits a higher voltage (1.16 V), indicating that the EP-based electrolyte will decompose at a higher voltage to form SEI layer. In the subsequent cycles,
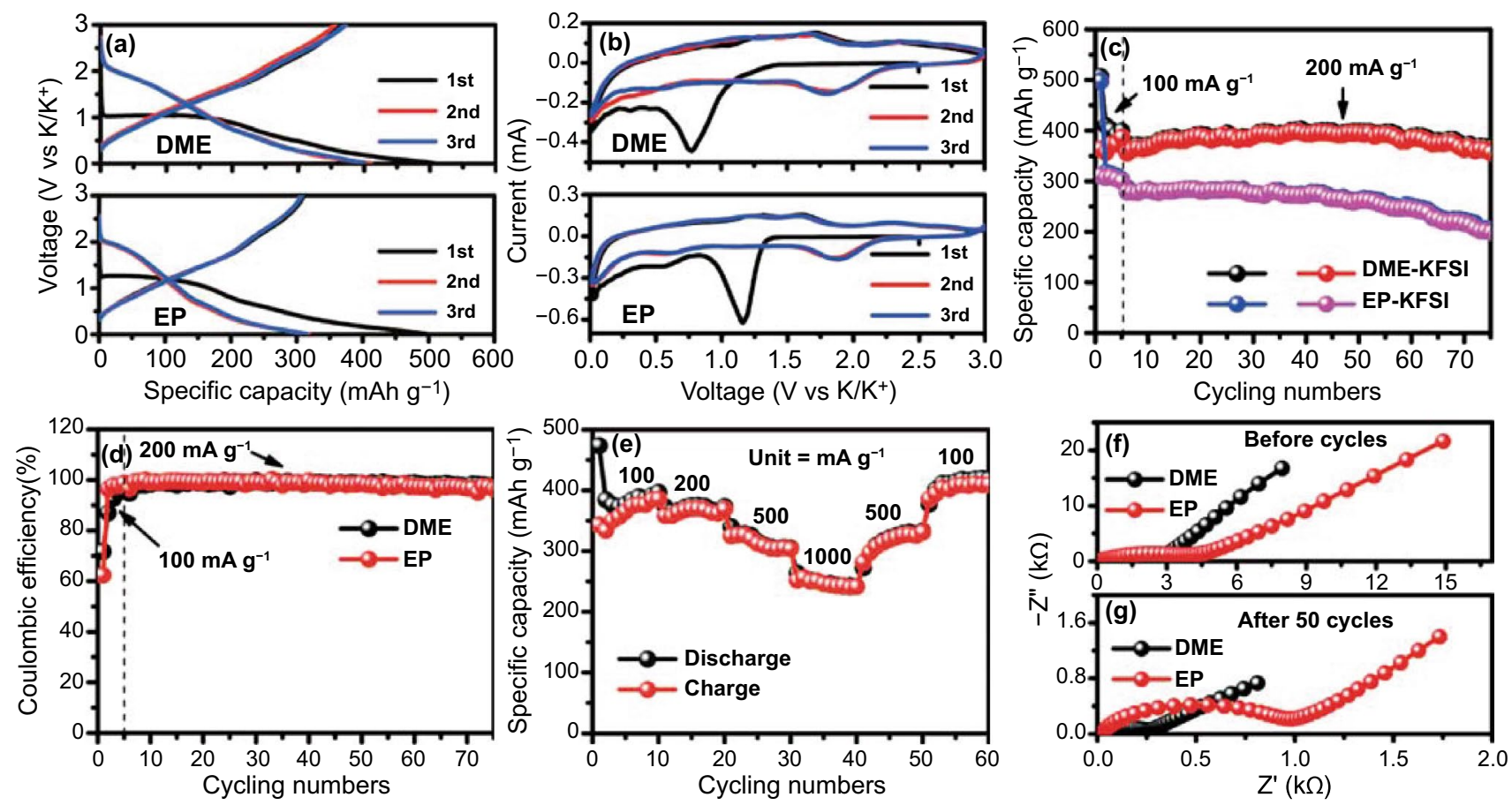

Fig. 2 a GCD curves, b CV curves, c cycling numbers and d Coulombic efficiencies of FeSe ${ }_{2} @$ RGO electrode with DME-based and EP-based electrolytes; e rate performance of $\mathrm{FeSe}_{2} @ \mathrm{RGO}$ electrode with DME-based electrolyte; EIS spectra of $\mathrm{FeSe}_{2} @ \mathrm{RGO}$ electrode with DME-based and EP-based electrolytes $\mathbf{f}$ before and $\mathbf{g}$ after 50 cycles 
all the CV curves exhibit similar shape, indicating that the batteries in both electrolytes exhibit the similar reaction process. Figure $2 \mathrm{c}$ shows the cycling performance of the batteries in DME-based and EP-based electrolytes. To facilitate the adequate electrochemical reaction of electrode material, we firstly measure the cycling performance at $100 \mathrm{~mA} \mathrm{~g}^{-1}$ after 5 cycles and then switched to the high current density of $200 \mathrm{~mA} \mathrm{~g}^{-1}$. We find that the $\mathrm{FeSe}_{2} @$ RGO matching EP-based electrolyte only delivers an initial discharge specific capacity of $497 \mathrm{mAh} \mathrm{g}^{-1}$ at $100 \mathrm{~mA} \mathrm{~g}^{-1}$ and keeps an initial reversible specific capacity of $310 \mathrm{mAh}$ $\mathrm{g}^{-1}$. After switching the current density to $200 \mathrm{~mA} \mathrm{~g}^{-1}$, our battery with EP-based electrolyte shows a distinct capacity decay to $198 \mathrm{mAh} \mathrm{g}^{-1}$ after 75 cycles. When changing the electrolyte to DME-based electrolyte, the electrode delivers a similar initial discharge specific capacity of $507 \mathrm{mAh}$ $\mathrm{g}^{-1}$ at $100 \mathrm{~mA} \mathrm{~g}^{-1}$. However, the initial reversible specific capacity of $364 \mathrm{mAh} \mathrm{g}^{-1}$ shows a significant improvement. Meanwhile, it is also found that the electrode in DME-based electrolyte receives a specific capacity of $356 \mathrm{mAh} \mathrm{g}^{-1}$ after 75 cycles when the current density converts to $200 \mathrm{~mA} \mathrm{~g}^{-1}$, which exhibits higher capacity stability compared with the electrode in EP-based electrolyte. Furthermore, an initial Coulombic efficiency (CE) of the electrode in DME-based electrolyte $(71.8 \%)$ can be observed, which is higher than that of electrode in EP-based electrolyte (62.4\%, Fig. 2d), indicating that DME-based electrolyte will promote the reduction in irreversible products $[41,42]$. To further investigate the electrochemical performance, we also present the rate performance of electrode in DME-based electrolyte and EP-based electrolyte, as shown in Figs. 2e and S6. It is found that our electrode in DME-based electrolyte delivers the reversible specific capacities of 386, 369, 304 and $242 \mathrm{mAh} \mathrm{g}^{-1}$ in the current densities of 100, 200, 500 and $1000 \mathrm{~mA} \mathrm{~g}^{-1}$, respectively, which is also higher than that of the electrode in EP-based electrolyte. With the recovery of current densities to 500 and $100 \mathrm{~mA} \mathrm{~g}^{-1}$, the reversible specific capacities also recover to 331 and $409 \mathrm{mAh}$ $\mathrm{g}^{-1}$, respectively. This result indicates that our electrode in DME-based electrolyte presents excellent reversibility. To analyze the influence of different electrolytes for K-ion storage, the EIS spectra of electrode with DME- and EP-based electrolytes before and after cycles are provided (Fig. 2f, g). A noticeably smaller impedance arises when the electrode matches the DME-based electrolyte after the cycles, indicating that DME-based electrolyte can facilitate the K-ion transmission in battery [43]. In addition, we also find that the impedances of both electrodes decrease after cycles, which should be due to the activated process of electrode, resulting in the enhancement of ion migration. Figure S7 shows the SEM images of electrode in DME- and EP-based electrolytes after cycles. It is found that the electrode presents flatness in DME-based electrolyte and a crack can be observed in the electrode using EP-based electrolyte, demonstrating that DME-based electrolyte contributes to the stability of electrode.

\subsection{Mechanical Properties Analysis}

To further analyze the influence of different electrolytes for $\mathrm{K}$-ion storage, the mechanical properties of both electrodes in DME- and EP-based electrolytes after cycles are provided. Figure 3a, b shows the AFM images of $\mathrm{FeSe}_{2} @$ RGO electrodes in different electrolytes, in which the average roughness of the electrodes using DME- and EP-based electrolytes can be calculated to be 40 and $73 \mathrm{~nm}$, respectively. This result indicates that DME-based electrolyte contributes to the stability of electrode during potassiation-depotassiation process. Figure $3 \mathrm{c}-\mathrm{f}$ presents the force-displacement curves and corresponding detailed force analysis of SEI layers. In general, representative force-displacement curves are obtained from the press-in and pull-out process of the samples' surface by the AFM tip. In the pull-in process, the AFM tip will instantaneously lose the force if the surface presents the fracture feature, which exhibits a sudden downward force. The force responses in DME-based electrolyte include linear elastic region by the response from electrode, indicating the existence of elastic surface of electrode [25, 44]. In contrast, the force responses in EP-based electrolyte include linear elastic region and slope change region. The slope change region was induced by perfectly plastic process or hardening process, possibly because of fracture and tip sliding [45]. The irreversible plastic deformation will ruin surface morphology, while elastic deformation contributes to reliving strain and maintaining integrity during potassiation-depotassiation process [25]. To quantify the mechanical properties, we calculate the Young's modulus values of electrodes using these types of electrolytes (Fig. 3g, h). An average Young's modulus value of 5.28 GPa of electrode using DME-based electrolyte can be received, which is 2.8 times larger than that of electrode using EP-based electrolyte (1.85 

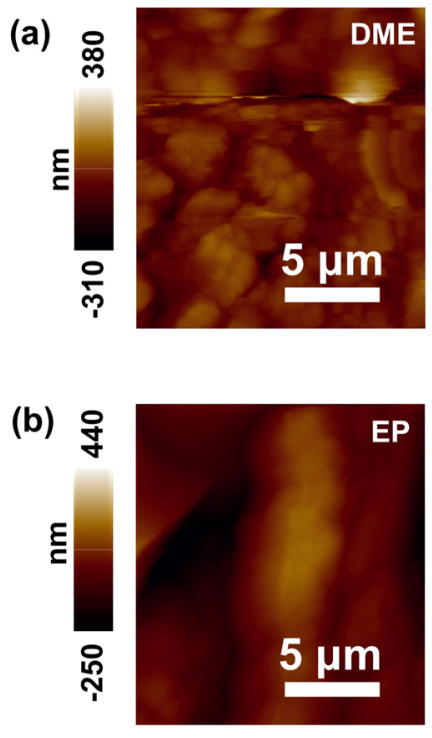
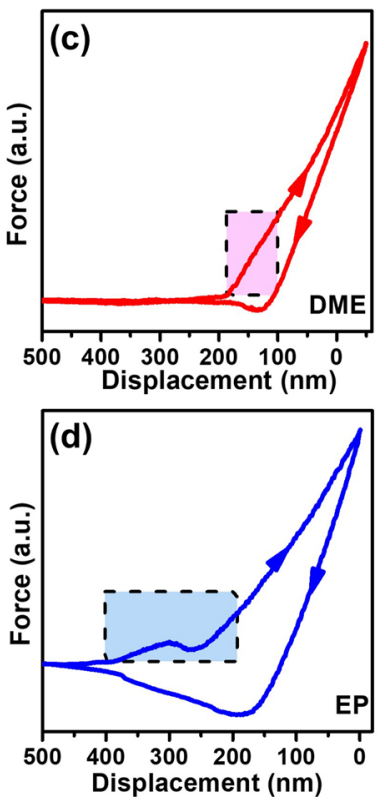
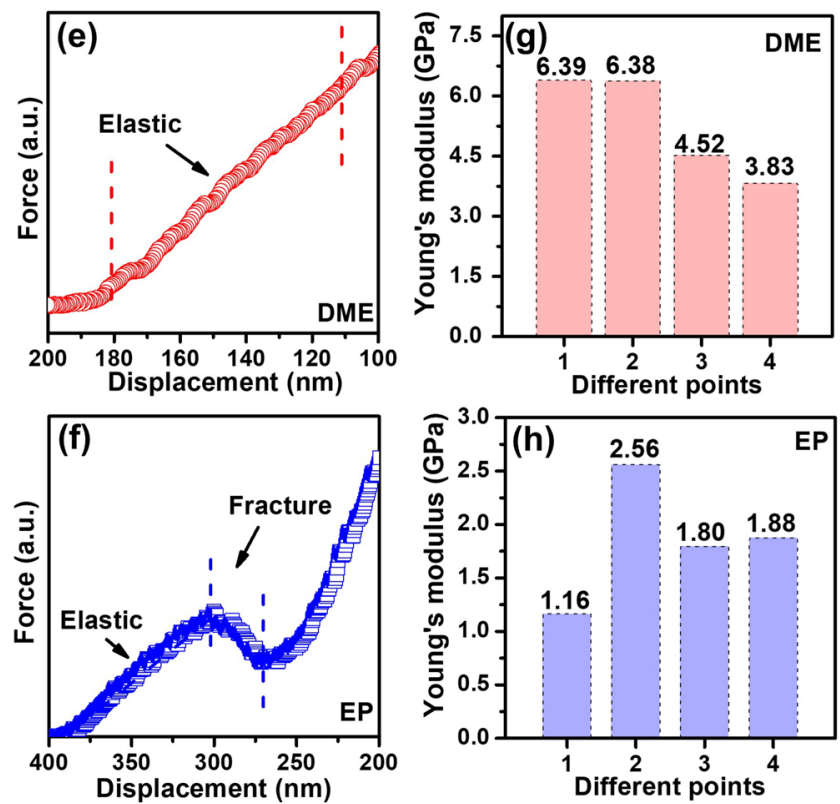

Fig. 3 AFM images of $\mathrm{FeSe}_{2} @$ RGO electrode using a DME-based and b EP-based electrolyte. Representative force-displacement curves $\mathrm{FeSe}_{2} @$ RGO electrode using c DME-based and d EP-based electrolyte after 50 cycles. A detailed force analysis of SEI layers using e DMEbased and $\mathbf{f}$ EP-based electrolytes after 50 cycles. Young's modulus of $\mathrm{FeSe}_{2} @ \mathrm{RGO}$ electrode using $\mathbf{g}$ DME-based and $\mathbf{h}$ EP-based electrolytes

GPa). Based on the great change of Young's modulus values, we suggest that the electrode will form compact and robust SEI layer in ether-based electrolyte. Such robust SEI layer is helpful for the inhibition of interfacial fracture, which facilitates the homogeneous electrochemical reaction for K-ion storage. On the contrary, lower Young's modulus values of electrode in EP-based electrolyte indicate the formation of loose and unstable SEI layer, which will cause the unstable performance of electrode during potassiation-depotassiation process [44].

\subsection{In situ Visualization Analysis}

To further monitor the K-ion storage enhancement of electrode in different electrolytes, we developed the in situ visualization technique to observe the reaction process in real time. Figure $4 \mathrm{a}-\mathrm{h}$ shows the visual images of electrode using DME-based electrolyte under different potassiation-depotassiation states in the first cycle. It is found that only a few particles on the electrode gradually strip together and the corresponding luster becomes slightly bright during the potassiation process. This phenomenon is due to the light reflection, which is caused by the volume expansion of the electrode during the insertion of K-ion. In the depotassiation process, it is found that the strip shape of electrode disappears and recovers to the original shape, indicating that our electrode presents an excellent reversibility. Generally, we consider that the expansion of the electrode material is closely related to the capacity in the same electrode. From the above results, the morphology of the electrode only presents a few minor changes, indicating that our electrode presents a homogeneous potassiation and depotassiation in DME-based electrolyte. According to the calculation in previous work, the K-ion presents relatively low energy change during migration process in the ether solvent, indicating that $\mathrm{K}$-ion can migrate into electrode more easily in ether-based electrolyte $[13,46]$. Therefore, we suggest that homogeneous potassiation and depotassiation process is mainly due to the high migration of K-ion in DME-based electrolyte. Furthermore, the homogeneous potassiation and depotassiation of electrode also provide less destruction of SEI layer, contributing to form a pyknotic SEI layer, which results in the high Young's modulus of SEI layer. In addition, the high Young's modulus of SEI layer also can resist the stress caused by local growth of electrode, which also facilitates the homogeneous potassiation and depotassiation reaction. To further confirm this result, we also provide the visual images of electrode using DME-based electrolyte under 

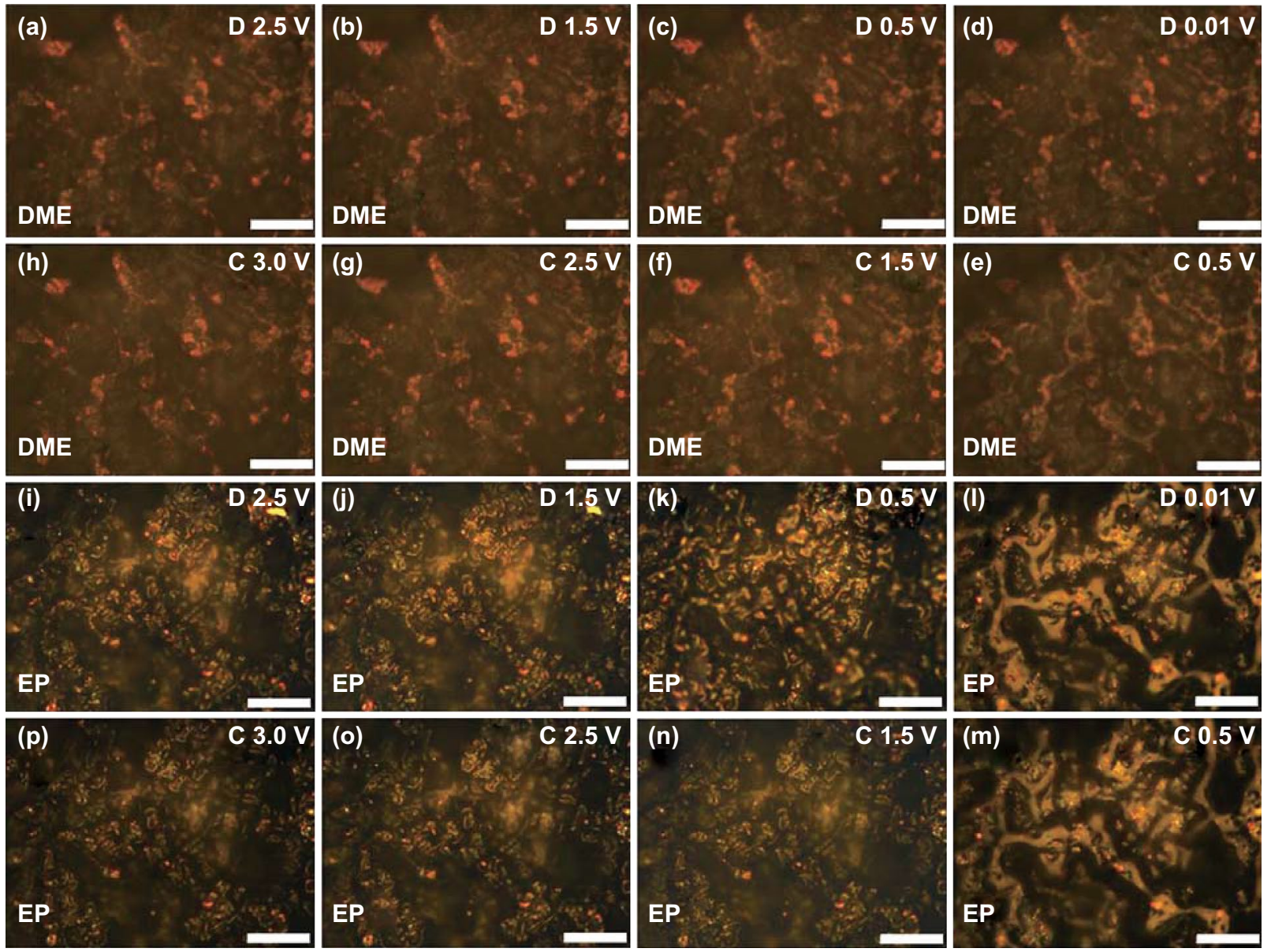

Fig. 4 In situ visualization: a-h different potassiation-depotassiation states of $\mathrm{FeSe}_{2} @ \mathrm{RGO}$ electrode using DME-based electrolyte in the first cycle; $\mathbf{i}-\mathbf{p}$ different potassiation-depotassiation states of $\mathrm{FeSe}_{2} @ \mathrm{RGO}$ electrode using EP-based electrolyte in the first cycle. The scale bar in each image is $50 \mu \mathrm{m}$

different potassiation-depotassiation states in the second cycle (Fig. S8a-h), which shows a similar result in the first cycle. Due to the homogeneous reaction of electrode in DME-based electrolyte, the integrity of electrode will be held during potassiation-depotassiation process, which contributes to the improvement in its cycling performance [47, 48]. When EP-based electrolyte is used (Fig. 4i-p), the phenomenon of morphologic change is even more drastic. Due to the relatively low migration of K-ion in carbonate solvent, a larger K-ion concentration difference occurs during potassiation-depotassiation process. This K-ion concentration difference will induce the inhomogeneous electrochemical reaction, resulting in the drastic morphologic change during potassiation-depotassiation process. In addition, the lower mechanical property of SEI layer in EP-based electrolyte may be beyond restraint excessive local stress, which also results in the enormous morphologic change. The visual images (Fig. S8i-p) in the second cycle also confirm the great variation in electrode during potassiation-depotassiation process. Due to the great variation in the subsequent cycles, the pulverization of electrode will be easier to occur, which results in the capacity decay of electrode. In addition, we also select the images of electrode in EP-based electrolyte with max variation during potassiation-depotassiation process for comparison, as shown in Fig. S9. Videos S1 and $\mathrm{S} 2$ show the whole reaction process of electrode using DME-based and EP-based electrolytes, respectively, in which the change of electrodes can be intuitively observed.

From the test of mechanical properties of SEI layer and the in situ visualization results of electrode, we raise the schematics to describe the expansion process of electrodes in DME-based and EP-based electrolytes, as shown in Fig. 5. 

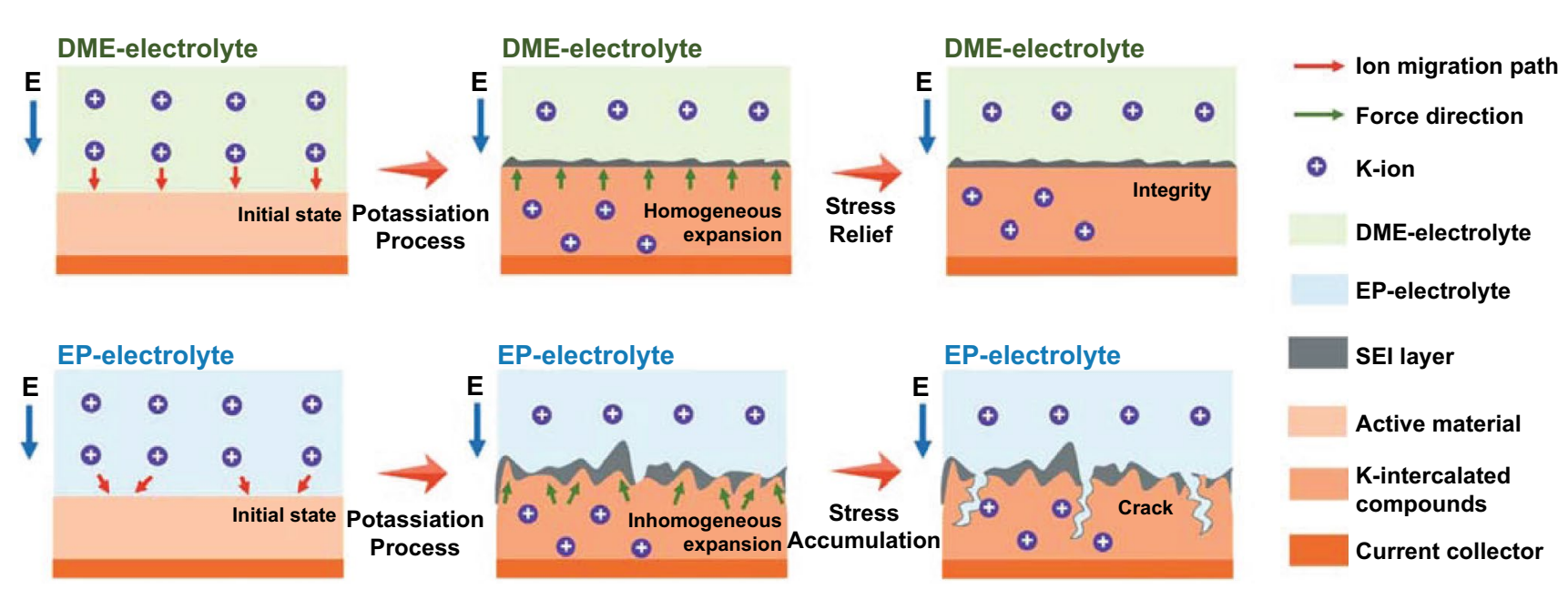

SEI layer

Fig. 5 Schematic illustration of expansion process of electrodes in DME- and EP-based electrolyte

Due to better ion mobility in ether-based electrolyte, it presents a shorter path for $\mathrm{K}$-ion migration, which facilitates a homogeneous volume expansion of $\mathrm{FeSe}_{2} @ \mathrm{RGO}$ electrode during the potassiation process. In addition, the robust SEI layer also provides a protection to control the expansion of the electrode material, which also results to the stress relief and realizes to integrity of electrode in subsequent potassiation-depotassiation process. If changing to EP-based electrolyte, it presents a longer path for K-ion migration due to the lower ion mobility in carbonate-based electrolyte. Therefore, an inhomogeneous volume expansion of electrode will occur, leading to a huge morphological change of electrode. Meanwhile, the low mechanical property of the SEI layer in EP electrolyte also results in the crack with the stress accumulation in subsequent potassiation-depotassiation process. This phenomenon will seriously damage the cyclic stability of electrode for K-ion storage.

\subsection{Dynamics Analysis}

Figure 6a, b shows the $\mathrm{CV}$ curves of $\mathrm{FeSe}_{2} @ \mathrm{RGO}$ electrode using DME-based and EP-based electrolyte in different scan rates after 5 cycles. All the $\mathrm{CV}$ curves exhibit the similar shape, but the CV curves of electrode in DME-based electrolyte present broad redox peaks. To investigate the change of CV curves, we analyzed their electrochemical behavior. The relationship between peak current $(i)$ and scan rate $(v)$ is determined by Eq. (1) $[49,50]$ : $i=a v^{b}$

which can be rearranged to Eq. (2):

$\log i=\log a+b \log v$

where $a$ and $b$ are the adjustable parameters and $b$ is confirmed by the slope in Eq. (2). Usually, the $b$ value closed to 0.5 is the diffusion-controlled behavior and the $b$ value closed to 1 indicates the capacitive behavior. In Fig. 6c, d, it is found that the $b$ values of all the peaks are closed to 1 , illustrating that the electrode in both electrolytes exhibits capacitive behavior. To quantify the electrochemical behavior, we calculate the capacitive contributions of electrode in both electrolytes according to Eq. (3) [51, 52]:

$i(V)=k_{1} v+k_{2} v^{1 / 2}$

which can be converted to Eq. (4):

$i(V) / v^{1 / 2}=k_{1} v^{1 / 2}+k_{2}$

where $k_{1} v$ and $k_{2} v^{1 / 2}$ are deemed as the capacitive and diffusion-controlled behaviors, respectively. According to Eq. (4), the electrode in DME-based electrolyte receives a capacitive contribution of $87 \%$ at a scan rate of $1 \mathrm{mV} \mathrm{s}^{-1}$ (Fig. 6e), which is higher than that of electrode in EP-based electrolyte (82\%, Fig. 6f). Figure 6g, h shows the capacitive contributions at different scan rates of electrode in DMEbased and EP-based electrolytes, respectively. The electrode in DME-based electrolyte delivers the capacitive contributions of $76 \%, 80 \%, 83 \%, 86 \%, 87 \%$ and $88 \%$ at the scan rates of $0.2,0.4,0.6,0.8,1.0$ and $1.2 \mathrm{mV} \mathrm{s}^{-1}$, respectively. Compared with the electrode in DME-based electrolyte, the 

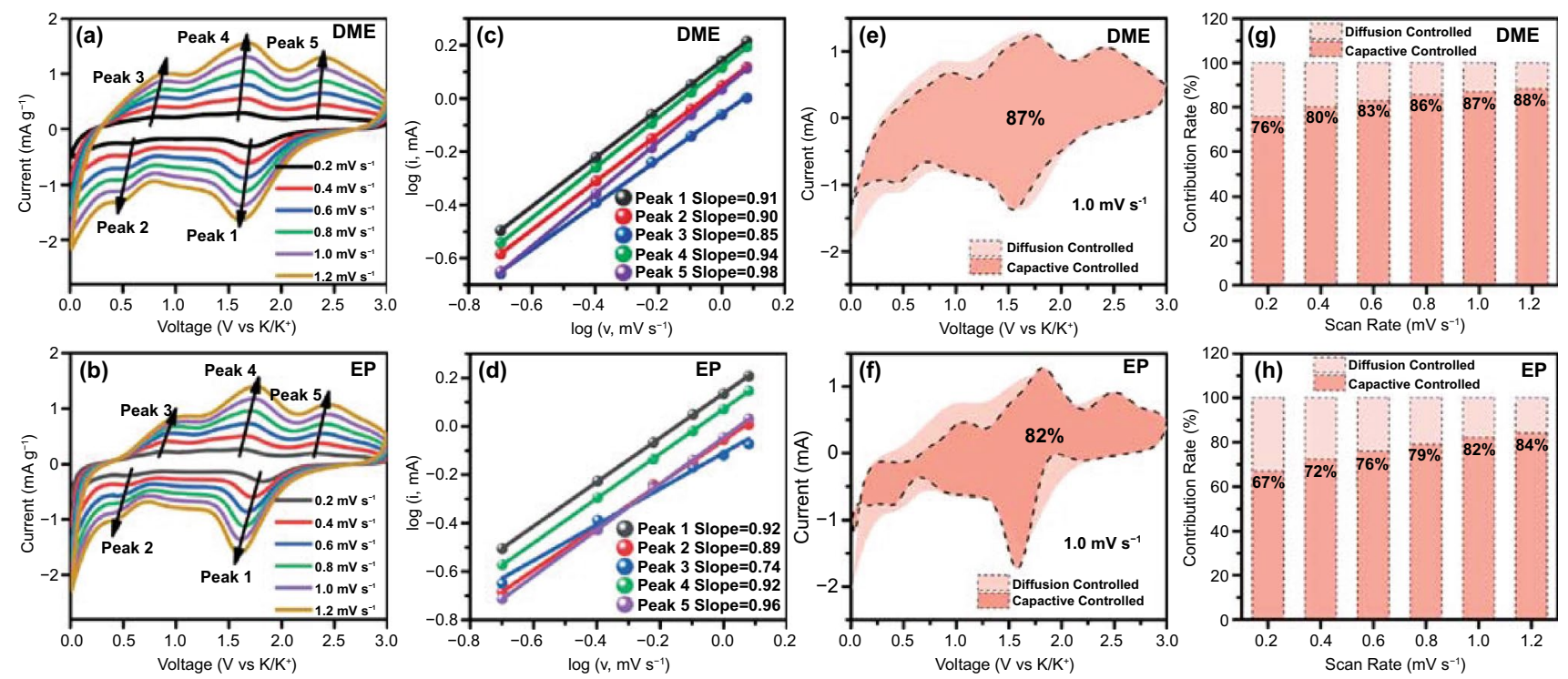

Fig. $6 \mathrm{CV}$ curves of $\mathrm{FeSe}_{2} @ \mathrm{RGO}$ electrode using a DME-based and b EP-based electrolyte in different scan rates. The relationship between log (i) and $\log (v)$ of $\mathrm{FeSe}_{2} @$ RGO electrode using c DME-based and d EP-based electrolytes. The capacitive contribution area of FeSe $\mathrm{C}_{2} \mathrm{RGO}$ electrode using e DME-based and $\mathbf{f}$ EP-based electrolytes at $1.0 \mathrm{mV} \mathrm{s}^{-1}$. Capacitive contribution ratios across different scan rates of FeSe $\mathrm{R}_{2} \mathrm{RGO}$ electrode using $\mathbf{g}$ DME-based and $\mathbf{h}$ EP-based electrolytes

electrode in EP-based electrolyte presents the lower capacitive contributions of $67 \%, 72 \%, 76 \%, 79 \%, 82 \%$ and $84 \%$ at the scan rates of $0.2,0.4,0.6,0.8,1.0$ and $1.2 \mathrm{mV} \mathrm{s}^{-1}$, respectively. According to this result, we suggest that the capacitive contribution of electrode is closely related to the electrolyte. In DME-based electrolyte, K-ion will present high ion migration rate, indicating that $\mathrm{K}$-ion can insert into electrode uniformly under the effect of electric field forces. The homogeneous insertion of K-ion contributes to the ion adsorption on the surface of electrode, which facilitates the improvement in capacitive contribution. Of course, the relatively higher capacitive contribution of electrode in DME-based electrolyte is also helpful for the improvement in cycling stability of electrode, which is consistent with the results of visualization measurement.

\subsection{In situ Raman Analysis}

Finally, we also provide in situ Raman spectra of electrode in DME-based electrolyte in Fig. 7 to further illustrate the potassiation-depotassiation process. Figure S10 displays the photograph of the practical in situ Raman spectra testing system. In the in situ Raman spectra, Fig. 7a shows the testing potassiation-depotassiation states according to the
$\mathrm{CV}$ curves. Figure $7 \mathrm{~b}, \mathrm{c}$ presents the corresponding Raman spectra of electrode in low wavenumbers region and high wavenumbers region, respectively. In the initial state, a distinct peak at $253 \mathrm{~cm}^{-1}$ could be detected, which is attributed to the intrinsic peak of $\mathrm{FeSe}_{2}$. With the potassiation process proceeding, this peak gradually disappears at $1.8 \mathrm{~V}$ due to the break of the original vibration mode of $\mathrm{FeSe}_{2}$ and a new peak at $213 \mathrm{~cm}^{-1}$ appears, indicating that $\mathrm{FeSe}_{2}$ transforms into intermediate. We also notice that all peaks disappear in complete potassiation, which is related to the loss of resonance by the K-ion intercalant [53]. During the depotassiation process, some new peaks at 146, 327, 398, 466 and $669 \mathrm{~cm}^{-1}$ arise, and we suggest that this phenomenon should be the structural remodeling of $\mathrm{FeSe}_{2}$ intermediate rather than the primary structure of $\mathrm{FeSe}_{2}$. In the high wavenumber region, D-band and G-band can be observed in initial stage. With the potassiation process proceeding, we can detect a slight change of intensity of D-band and G-band. The intensity of G-band increases after completely depotassiation compared with that in the initial stage, indicating the formation of more orderliness of carbon in electrode, which is due to the realignment of interlayer distance [54]. 

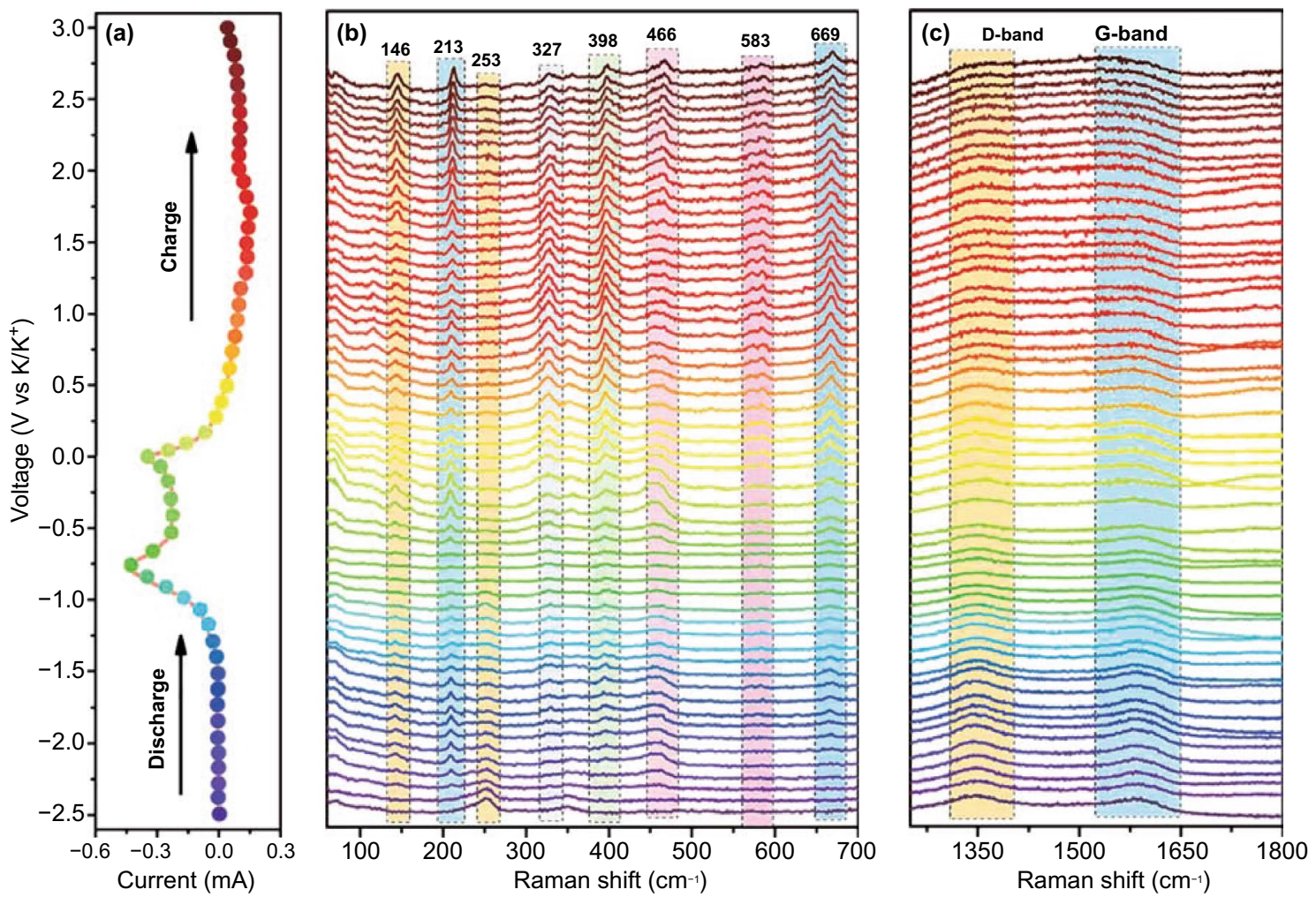

Fig. 7 In situ Raman spectra of $\mathrm{FeSe}_{2} @ \mathrm{RGO}$ electrode using DME-based electrolyte in different potassiation-depotassiation states: a the corresponding voltage position; in situ Raman spectra at $\mathbf{b}$ low wavenumbers and $\mathbf{c}$ high wavenumbers

\section{Conclusions}

In this work, we rationally obtained iron selenide composite cooperated with different electrolytes for KIBs and the results show that the electrode matching ether-based (DME) electrolyte presents a reversible specific capacity of $356 \mathrm{mAh} \mathrm{g}^{-1}$ at $200 \mathrm{~mA} \mathrm{~g}^{-1}$ after 75 cycles, which is better than that cooperated with carbonate-based (EP) electrolyte. To investigate the electrochemical enhancement mechanism of KIBs, we also utilized in situ visualization technique and Raman spectra to monitor their potassiation-depotassiation process. The result shows that iron selenide presents less volume expansion in ether-based electrolyte. According to the test of mechanical properties of the electrode in both electrolytes, an elastic interphase layer in ether-based electrolyte can be detected, which also contributes to the improvement in reversibility and stability for KIBs and is consistent with the results from the in situ characteristics.
Acknowledgements The authors thank the financial supports from the National Natural Science Foundation of China (51772135), the Fundamental Research Funds for the Central Universities (11619103, 21621406), the Science and Technology Program of Guangzhou, China (202102020737, 201605030008) and the Shenzhen Science and Technology Program (JCYJ20200109113606007). We also thank Prof. Weiguang Xie and Dr. Haojie Lai for the measurement of AFM.

Open Access This article is licensed under a Creative Commons Attribution 4.0 International License, which permits use, sharing, adaptation, distribution and reproduction in any medium or format, as long as you give appropriate credit to the original author(s) and the source, provide a link to the Creative Commons licence, and indicate if changes were made. The images or other third party material in this article are included in the article's Creative Commons licence, unless indicated otherwise in a credit line to the material. If material is not included in the article's Creative Commons licence and your intended use is not permitted by statutory regulation or exceeds the permitted use, you will need to obtain permission directly from the copyright holder. To view a copy of this licence, visit http://creativecommons.org/licenses/by/4.0/.

Supplementary Information The online version contains supplementary material available at https://doi.org/10.1007/ s40820-021-00708-1. 


\section{References}

1. L. Li, R. Zhao, D. Pan, S. Yi, L. Gao et al., Constructing trifunctional modification for spinel $\mathrm{LiNi}_{0.5} \mathrm{Mn}_{1.5} \mathrm{O}_{4}$ via fast ion conductor. J. Power Sources 450, 227677 (2020). https://doi. org/10.1016/j.jpowsour.2019.227677

2. W.W. Jiang, Q.H. Liu, J.F. Peng, Y.H. Jiang, Y.H. Ding et al., $\mathrm{Co}_{9} \mathrm{~S}_{8}$ nanoparticles embedded into amorphous carbon as anode materials for lithium-ion batteries. Nanotechnology 31, 235713 (2020). https://doi.org/10.1088/1361-6528/ab7887

3. N.F. Zhou, W. Qin, C. Wu, C.K. Jia, Graphene-attached vanadium sulfide composite prepared via microwave-assisted hydrothermal method for high performance lithium ion batteries. J. Alloys Compd. 834, 155073 (2020). https://doi.org/ 10.1016/j.jallcom.2020.155073

4. L. Li, R. Zhao, T.H. Xu, D.D. Wang, D. Pan et al., Stabilizing a high-voltage $\mathrm{LiNi}_{0.5} \mathrm{Mn}_{1.5} \mathrm{O}_{4}$ cathode towards all solid state batteries: a Li-Al-Ti-P-O solid electrolyte nano-shell with a host material. Nanoscale 11, 8967-8977 (2019). https://doi. org/10.1039/C9NR01655D

5. T. Sun, Z.-J. Li, X. Yang, S. Wang, Y.-H. Zhu et al., Iminerich poly(o-phenylenediamine) as high-capacity trifunctional organic electrode for alkali-ion batteries. CCS Chem. 1, 365372 (2019). https://doi.org/10.31635/ccschem.019.20190003

6. C. Yang, S. Xin, L.Q. Mai, Y. You, Materials design for highsafety sodium-ion battery. Adv. Energy Mater. 11, 2000974 (2021). https://doi.org/10.1002/aenm.202000974

7. X. Yuan, S. Chen, J. Li, J. Xie, G. Yan et al., Understanding the improved performance of sulfur-doped interconnected carbon microspheres for Na-ion storage. Carbon Energy (2021). https://doi.org/10.1002/cey2.98

8. W.C. Zhang, Y.J. Liu, Z.P. Guo, Approaching high-performance potassium-ion batteries via advanced design strategies and engineering. Sci. Adv. 5, eaav7412 (2019). https://doi.org/ 10.1126/sciadv.aav7412

9. M. Sha, L. Liu, H. Zhao, Y. Lei, Anode materials for potassium-ion batteries: current status and prospects. Carbon Energy 2, 350-369 (2020). https://doi.org/10.1002/cey2.57

10. L. Ma, J. Li, T. Wu, P. Sun, S. Tan et al., Re-oxidation reconstruction process of solid electrolyte interphase layer derived from highly active anion for potassium-ion batteries. Nano Energy 87, 106150 (2021). https://doi.org/10.1016/j.nanoen. 2021.106150

11. J. Zheng, Y. Yang, X.L. Fan, G.B. Ji, X. Ji et al., Extremely stable antimony-carbon composite anodes for potassium-ion batteries. Energy Environ. Sci. 12, 615-623 (2019). https:// doi.org/10.1039/C8EE02836B

12. J.L. Li, N. Zhuang, J.P. Xie, Y.Q. Zhu, H.J. Lai et al., Carboxymethyl cellulose binder greatly stabilizes porous hollow carbon submicrospheres in capacitive K-ion storage. ACS Appl. Mater. Interfaces 11, 15581-15590 (2019). https://doi.org/10. 1021/acsami.9b02060

13. J.L. Li, N. Zhuang, J.P. Xie, X.D. Li, W.C. Zhuo et al., K-ion storage enhancement in $\mathrm{Sb}_{2} \mathrm{O}_{3}$ /reduced graphene oxide using ether-based electrolyte. Adv. Energy Mater. 10, 1903455 (2020). https://doi.org/10.1002/aenm.201903455
14. E.J. Zhang, X.N. Jia, B. Wang, J. Wang, X.Z. Yu, B.G. Lu, Carbon dots@rGO paper as freestanding and flexible potassium-ion batteries anode. Adv. Sci. 7, 2000470 (2020). https:// doi.org/10.1002/advs.202000470

15. X.D. Li, J.L. Li, L. Ma, C.Y. Yu, Z. Ji et al., Graphite anode for potassium ion batteries: current status and perspective. Energy Environ. Mater. (2021). https://doi.org/10.1002/eem2.12194

16. J.Y. Cheng, L.F. Gao, T. Li, S. Mei, C. Wang et al., Twodimensional black phosphorus nanomaterials: emerging advances in electrochemical energy storage science. Nano-Micro Lett. 12, 179 (2020). https://doi.org/10.1007/ s40820-020-00510-5

17. K.Z. Cao, H.Q. Liu, W.Y. Li, Q.Q. Han, Z. Zhang et al., CuO nanoplates for high-performance potassium-ion batteries. Small 15, 1901775 (2019). https://doi.org/10.1002/smll.20190 1775

18. L. Zhang, X. Gu, X. Mao, S. Wen, P. Dai et al., Boosting fast and stable potassium storage of iron selenide/carbon nanocomposites by electrolyte salt and solvent chemistry. J. Power Sources 486, 229373 (2021). https://doi.org/10.1016/j.jpows our.2020.229373

19. Q. Yao, C. Zhu, Advanced Post-potassium-ion batteries as emerging potassium-based alternatives for energy storage. Adv. Funct. Mater. 30, 2005209 (2020). https://doi.org/10. 1002/adfm.202005209

20. H. Min, M. Li, H. Shu, X. Zhang, T. Hu et al., FeSe ${ }_{2}$ nanoparticle embedded in 3D honeycomb-like $\mathrm{N}$-doped carbon architectures coupled with electrolytes engineering boost superior potassium ion storage. Electrochim. Acta 366, 137381 (2021). https://doi.org/10.1016/j.electacta.2020.137381

21. Q.C. Pan, M. Zhang, L.X. Zhang, Y.H. Li, Y. Li et al., $\mathrm{FeSe}_{2} @ \mathrm{C}$ microrods as a superior long-life and high-rate anode for sodium ion batteries. ACS Nano 14, 17683-17692 (2020). https://doi.org/10.1021/acsnano.0c08818

22. Y.Z. Liu, C.H. Yang, Y.P. Li, F.H. Zheng, Y.J. Li et al., $\mathrm{FeSe}_{2} /$ nitrogen-doped carbon as anode material for potassium-ion batteries. Chem. Eng. J. 393, 124590 (2020). https://doi.org/ 10.1016/j.cej.2020.124590

23. W. Zhao, Q.W. Tan, K. Han, D.L. He, P. Li et al., Achieving fast and stable lithium/potassium storage by in situ decorating $\mathrm{FeSe}_{2}$ nanodots into three-dimensional hierarchical porous carbon networks. J. Phys. Chem. C 124, 12185-12194 (2020). https://doi.org/10.1021/acs.jpcc.9b11432

24. T.X. Wang, W.T. Guo, G. Wang, H. Wang, J.T. Bai et al., Highly dispersed $\mathrm{FeSe}_{2}$ nanoparticles in porous carbon nanofibers as advanced anodes for sodium and potassium ion batteries. J. Alloys Compd. 834, 155265 (2020). https://doi. org/10.1016/j.jallcom.2020.155265

25. J.P. Xie, X.D. Li, H.J. Lai, Z.J. Zhao, J.L. Li et al., A robust solid electrolyte interphase layer augments the ion storage capacity of bimetallic-sulfide-containing potassium-ion batteries. Angew. Chem. Int. Ed. 58, 14740-14747 (2019). https:// doi.org/10.1002/anie.201908542

26. L. Zhou, Z. Cao, W. Wahyudi, J. Zhang, J.Y. Hwang et al., Electrolyte engineering enables high stability and capacity alloying anodes for sodium and potassium ion batteries. ACS 
Energy Lett. 5, 766-776 (2020). https://doi.org/10.1021/acsen ergylett.0c00148

27. H. Wang, D.D. Yu, X. Wang, Z.Q. Niu, M.X. Chen et al., Electrolyte chemistry enables simultaneous stabilization of potassium metal and alloying anode for potassium-ion batteries. Angew. Chem. Int. Ed. 58, 16451-16455 (2019). https:// doi.org/10.1002/anie.201908607

28. J. Wu, Q. Zhang, S. Liu, J. Long, Z. Wu et al., Synergy of binders and electrolytes in enabling microsized alloy anodes for high performance potassium-ion batteries. Nano Energy 77, 105118 (2020). https://doi.org/10.1016/j.nanoen.2020. 105118

29. K. Lei, C. Wang, L. Liu, Y. Luo, C. Mu et al., A porous network of bismuth used as the anode material for high-energydensity potassium-ion batteries. Angew. Chem. Int. Ed. 57, 4687-4691 (2018). https://doi.org/10.1002/anie.201801389

30. K. Han, W. Zhao, Q. Yu, Z. Liu, P. Li et al., Marcasite-FeS @ carbon nanodots anchored on 3D cell-like graphenic matrix for high-rate and ultrastable potassium ion storage. J. Power Sources 469, 228429 (2020). https://doi.org/10.1016/j.jpows our.2020.228429

31. Q.G. Pan, Y.P. Zheng, Z.P. Tong, L. Shi, Y.B. Tang, Novel lamellar tetrapotassium pyromellitic organic for robust highcapacity potassium storage. Angew. Chem. Int. Ed. 60, 1183511840 (2021). https://doi.org/10.1002/anie.202103052

32. X.Q. Chang, X.L. Zhou, X.W. Ou, C.S. Lee, J.W. Zhou et al., Ultrahigh nitrogen doping of carbon nanosheets for high capacity and long cycling potassium ion storage. Adv. Energy Mater. 9, 1902672 (2019). https://doi.org/10.1002/aenm.20190 2672

33. K. Yang, Q.R. Liu, Y.P. Zheng, H. Yin, S.Q. Zhang et al., Locally ordered graphitized carbon cathodes for high-capacity dual-ion batteries. Angew. Chem. Int. Ed. 60, 6326-6332 (2021). https://doi.org/10.1002/anie.202016233

34. J.L. Li, W. Qin, J.P. Xie, H. Lei, Y.Q. Zhu et al., Sulphurdoped reduced graphene oxide sponges as high-performance free-standing anodes for K-ion storage. Nano Energy 53, 415424 (2018). https://doi.org/10.1016/j.nanoen.2018.08.075

35. J. Xie, Y. Zhu, N. Zhuang, H. Lei, W. Zhu et al., Rational design of metal organic framework-derived $\mathrm{FeS}_{2}$ hollow nanocages@reduced graphene oxide for K-ion storage. Nanoscale 10, 17092-17098 (2018). https://doi.org/10.1039/ C8NR05239E

36. Y. Tian, Z.Y. Wang, J.M. Fu, K.Q. Xia, J.G. Lu et al., FeSe $/$ carbon nanotube hybrid lithium-ion battery for harvesting energy from triboelectric nanogenerators. Chem. Commun. 55, 10960-10963 (2019). https://doi.org/10.1039/C9CC0 $5069 \mathrm{H}$

37. S.K. Jiang, M.J. Xiang, J.Y. Zhang, S.Q. Chu, A. Marcelli et al., Rational design of hierarchical $\mathrm{FeSe}_{2}$ encapsulated with bifunctional carbon cuboids as an advanced anode for sodiumion batteries. Nanoscale 12, 22210-22216 (2020). https://doi. org/10.1039/D0NR06359B

38. Q. Zhang, R. Karthick, X.L. Zhao, L.G. Zhang, Y.M. Shi et al., $\mathrm{Sb}$ nanoparticle decorated rGO as a new anode material in aqueous chloride ion batteries. Nanoscale 12, 12268-12274 (2020). https://doi.org/10.1039/D0NR00862A

39. M. Yousaf, Z. Wang, Y. Wang, Y. Chen, U. Ali et al., Coreshell $\mathrm{FeSe}_{2} / \mathrm{C}$ nanostructures embedded in a carbon framework as a free standing anode for a sodium ion battery. Small 16, 2002200 (2020). https://doi.org/10.1002/smll.202002200

40. J. Ge, B. Wang, J. Wang, Q. Zhang, B. Lu, Nature of $\mathrm{FeSe}_{2} / \mathrm{N}-\mathrm{C}$ anode for high performance potassium ion hybrid capacitor. Adv. Energy Mater. 10, 1903277 (2020). https://doi. org/10.1002/aenm.201903277

41. C.Z. Zhang, F. Wang, F. Han, H. Wu, F.Q. Zhang et al., Improved electrochemical performance of sodium/potassium-ion batteries in ether-based electrolyte: cases study of $\mathrm{MoS}_{2} @ \mathrm{C}$ and $\mathrm{Fe}_{7} \mathrm{~S}_{8} @ \mathrm{C}$ anodes. Adv. Mater. Interfaces 7, 2000486 (2020). https://doi.org/10.1002/admi.202000486

42. K.X. Lei, F.J. Li, C.N. Mu, J.B. Wang, Q. Zhao et al., High K-storage performance based on the synergy of dipotassium terephthalate and ether-based electrolytes. Energy Environ. Sci. 10, 552-557 (2017). https://doi.org/10.1039/C6EE0 3185D

43. L. Ran, B. Luo, I.R. Gentle, T. Lin, Q. Sun et al., Biomimetic $\mathrm{Sn}_{4} \mathrm{P}_{3}$ anchored on carbon nanotubes as an anode for highperformance sodium-ion batteries. ACS Nano 14, 8826-8837 (2020). https://doi.org/10.1021/acsnano.0c03432

44. J.Q. Huang, X.Y. Guo, X.Q. Du, X.Y. Lin, J.Q. Huang et al., Nanostructures of solid electrolyte interphases and their consequences for microsized $\mathrm{Sn}$ anodes in sodium ion batteries. Energy Environ. Sci. 12, 1550-1557 (2019). https://doi.org/ 10.1039/C8EE03632B

45. T. Chen, F. Meng, Z. Zhang, J. Liang, Y. Hu et al., Stabilizing lithium metal anode by molecular beam epitaxy grown uniform and ultrathin bismuth film. Nano Energy 76, 105068 (2020). https://doi.org/10.1016/j.nanoen.2020.105068

46. Q. Xu, T. Chen, Z.G. Wu, Y.H. Liu, L. Qiu et al., General synthesis of $M_{x} S(M=C o, C u)$ hollow spheres with enhanced sodium-ion storage property in ether-based electrolyte. Ind. Eng. Chem. Res. 59, 1568-1577 (2020). https://doi.org/10. 1021/acs.iecr.9b06023

47. L.P. Wang, J.Y. Yang, J. Li, T. Chen, S.L. Chen et al., Graphite as a potassium ion battery anode in carbonate-based electrolyte and ether-based electrolyte. J. Power Sources 409, 24-30 (2019). https://doi.org/10.1016/j.jpowsour.2018.10.092

48. Y.E. Zhu, L.P. Yang, X.L. Zhou, F. Li, J.P. Wei et al., Boosting the rate capability of hard carbon with an ether-based electrolyte for sodium ion batteries. J. Mater. Chem. A 5, 9528-9532 (2017). https://doi.org/10.1039/C7TA02515G

49. X.D. Li, Z.B. Liu, J.L. Li, H. Lei, W.C. Zhuo et al., Insights on the mechanism of Na-ion storage in expanded graphite anode. J. Energy Chem. 53, 56-62 (2021). https://doi.org/10.1016/j. jechem.2020.05.022

50. Y. Wang, Y. Zhang, J. Shi, X. Kong, X. Cao et al., Tin sulfide nanoparticles embedded in sulfur and nitrogen dual-doped mesoporous carbon fibers as high-performance anodes with battery-capacitive sodium storage. Energy Storage Mater. 18, 366-374 (2019). https://doi.org/10.1016/j.ensm.2018.08.014 
51. W. Xiong, J.D. Zhang, Y. Xiao, Y.H. Zhu, Z.Y. Wang et al., Oxygen-rich nanoflake-interlaced carbon microspheres for potassium-ion battery anodes. Chem. Commun. 56, 34333436 (2020). https://doi.org/10.1039/D0CC00357C

52. R.C. Cui, B. Xu, H.J. Dong, C.C. Yang, Q. Jiang, N/O dualdoped environment-friendly hard carbon as advanced anode for potassium-ion batteries. Adv. Sci. 7, 1902547 (2020). https://doi.org/10.1002/advs.201902547

53. W. Tang, B.M. Goh, M.Y. Hu, C. Wan, B.B. Tian et al., In situ Raman and nuclear magnetic resonance study of trapped lithium in the solid electrolyte interface of reduced graphene oxide. J. Phys. Chem. C 120, 2600-2608 (2016). https://doi. org/10.1021/acs.jpcc.5b12551

54. J. Xie, J. Li, X. Li, H. Lei, W. Zhuo et al., Ultrahigh "relative energy density" and mass loading of carbon cloth anodes for K-ion batteries. CCS Chem. 2, 791-799 (2020). https://doi. org/10.31635/ccschem.020.202000203 